\title{
WHO assists Middle East authorities with coronavirus investigation
}

Published online: 05 December 2012

(C) Springer Healthcare 2012

medwireNews: The World Health Organization (WHO) has sent a team to Jordan at the request of the country's Ministry of Health to help investigate the emergence of a novel coronavirus that resulted in the deaths of two people earlier this year, and to assist with further epidemiologic surveillance.

As of November 30, 2012, the number of laboratory-confirmed cases of the new virus has risen to nine, since the original two cases were reported in Jordan in April 2012. In addition to the two original cases in Jordan, both of which were fatal, five cases (three deaths) have been reported in Saudi Arabia and two cases in Qatar.

There have been fears that the new coronavirus may be similar to the Severe Acute Respiratory Syndrome (SARS) virus, as they are distantly related, but current evidence suggests that the new virus is not easily transferable from person to person. However, the two small clusters of infections in Saudi Arabia and Jordan suggest that limited human-to-human transmission might be possible.

The updated WHO recommendations, published on December 3, 2012, suggest that people who have an unidentified acute respiratory infection, which may include fever and cough, who are suspected to have pneumonia or Acute Respiratory Distress Syndrome, and who either live in the Arabian Peninsula or have travelled there within the previous 10 days should be investigated and tested for possible infection with the new coronavirus. Laboratory assays for the virus have been developed and are available for this purpose.

The WHO guidelines also suggest that any contacts or healthcare workers who fall ill after extended contact with patients suspected to have the new coronavirus should be tested.

In addition to the acute respiratory symptoms associated with this virus, five of the nine patients developed acute renal failure.

The WHO and local authorities have been closely monitoring the situation and are trying to determine the source of the infection and the route of exposure of those infected. No animal-tohuman link has been established to date, and the small number of cases suggests that the virus does not easily transfer from person to person. 
Therefore, based on current data, the WHO does not advise special screening at border points, nor does it recommend any travel or trade restrictions be put in place at present.

By Helen Albert, Senior medwireNews Reporter

\section{Reference}

World Health Organization 